\title{
Experimental quantum learning of a spectral decomposition
}

\author{
Michael R. Geller $\odot,{ }^{1}$ Zoë Holmes, ${ }^{2}$ Patrick J. Coles,${ }^{3,4}$ and Andrew Sornborger ${ }^{2,4, *}$ \\ ${ }^{1}$ Center for Simulational Physics, University of Georgia, Athens, Georgia 30602, USA \\ ${ }^{2}$ Information Sciences, Los Alamos National Laboratory, Los Alamos, New Mexico 87545, USA \\ ${ }^{3}$ Theoretical Division, Los Alamos National Laboratory, Los Alamos, New Mexico 87545, USA \\ ${ }^{4}$ Quantum Science Center, Oak Ridge, Tennessee, 37931, USA
}

(Received 9 April 2021; accepted 7 July 2021; published 30 August 2021)

\begin{abstract}
Currently available quantum hardware allows for small-scale implementations of quantum machine learning algorithms. Such experiments aid the search for applications of quantum computers by benchmarking the nearterm feasibility of candidate algorithms. Here we demonstrate the quantum learning of a two-qubit unitary by a sequence of three parameterized quantum circuits containing a total of 21 variational parameters. Moreover, we variationally diagonalize the unitary to learn its spectral decomposition, i.e., its eigenvalues and eigenvectors. We illustrate how this can be used as a subroutine to compress the depth of dynamical quantum simulations. One can view our implementation as a demonstration of entanglement-enhanced machine learning, as only a single (entangled) training data pair is required to learn a $4 \times 4$ unitary matrix.
\end{abstract}

DOI: 10.1103/PhysRevResearch.3.033200

\section{INTRODUCTION}

Quantum simulation and machine learning are among the most promising applications of large-scale quantum computers. Of these, the discovery of algorithms with provable exponential speedup has been more challenging in the machine learning domain, in part because it is harder to port established machine learning techniques to the quantum setting [1]. Notable exceptions include linear system solvers [2-4], kernel methods [5,6], and Boltzmann machines [7,8]. But quantum simulation demonstrations [9-13] appear to be ahead of machine learning [14-20] in terms of the maximum problem sizes achieved, suggesting that quantum simulation might yield the earliest applications with quantum advantage.

Variational quantum algorithms [21-23] will likely facilitate near-term implementations for these applications. Such algorithms employ a problem-specific cost function that is evaluated on a quantum computer, while a classical optimizer trains a parameterized quantum circuit to minimize this cost.

Some variational quantum algorithms have interest beyond their ability to achieve quantum advantage on their own and can serve as subroutines for larger quantum algorithms. These include quantum autoencoders for data compression [24,25] and linear algebra methods [26-28]. A common subroutine is the training of a variational quantum state to approximate the ground state of a given $n$-qubit Hamiltonian, which can be the Hamiltonian of a simulated model [29] or some other opti-

\footnotetext{
*sornborg@lanl.gov

Published by the American Physical Society under the terms of the Creative Commons Attribution 4.0 International license. Further distribution of this work must maintain attribution to the author(s) and the published article's title, journal citation, and DOI.
}

mization objective [30-32]. Variational quantum algorithms to learn and diagonalize density matrices have also been developed [33-35], which is a fundamental subroutine that will have many uses including principal component analysis and estimation of quantum information quantities [36,37]. Another subroutine is the learning of one quantum state by a second state, where the output of a variational circuit is optimized to maximize the overlap with an input state that might itself be the output of another algorithm [38-41]. Although a minimum of $2\left(2^{n}-1\right)$ real parameters are required to do this exactly in general, it is widely believed that for many cases of interest a polynomial number of parameters will suffice.

Beyond learning states, it is also useful to variationally learn a unitary channel $\rho \mapsto U \rho U^{\dagger}$. This is more challenging because now all of the $4^{n}-1$ matrix elements must match for an exact replica $V$ of an arbitrary $U$, up to a global phase. A hybrid protocol for learning a unitary is provided by the quantum-assisted quantum compiling algorithm [38]. This is a low-depth subroutine appropriate for both near-term and fault-tolerant quantum computing.

Given a target unitary $U$ and parameterized unitary $V(\theta)$, both acting on $n$ qubits, quantum-assisted quantum compiling uses a maximally entangled Bell state on $2 n$ qubits to compute the Hilbert-Schmidt inner product, $\left|\operatorname{Tr}\left(U V(\theta)^{\dagger}\right)\right|$. Since this inner product is directly related to the average fidelity between states acted on by $U$ and $V(\theta)[42,43]$, this allows the action of $U$ on all possible input states to be studied using a single entangled input state. Consequently, with this entanglementenhanced learning strategy, only a single training state is needed to fully learn $U$, in contrast to the $\approx 2^{n}$ input-output pairs that are required in the absence of entanglement [44,45].

Although more challenging than state learning, learning a unitary can be used for a wide variety of quantum information applications, including circuit depth compression, 
noise-tailoring, benchmarking, and the "black box uploading" of an unknown experimental system unitary [38]. Quantumassisted quantum compiling has been demonstrated on $1+1$ qubits, where a single-qubit variational circuit $V(\theta)$ learned the value of a single-qubit unitary $U$ [38].

Quantum-assisted quantum compiling can be generalized to learn not only a unitary, but also its Schur decomposition $W(\theta) D(\gamma) W(\theta)^{\dagger}$, where $W(\theta)$ is a parameterized unitary and $D(\gamma)$ is a parameterized diagonal unitary. That is, one can use quantum-assisted quantum compiling to variationally diagonalize a unitary. This is useful for a variety of quantum information science applications, since access to the spectral decomposition of a unitary $U$ enables arbitrary powers of $U$ to be implemented using a fixed depth circuit. Specifically, suppose we learn the optimal parameters $\theta_{\text {opt }}$ and $\gamma_{\text {opt }}$ such that $U=W\left(\theta_{\text {opt }}\right) D\left(\gamma_{\text {opt }}\right) W\left(\theta_{\text {opt }}\right)^{\dagger}$. We can then implement $U^{k}$ using the fixed depth circuit $W\left(\theta_{\text {opt }}\right) D\left(k \gamma_{\text {opt }}\right) W\left(\theta_{\text {opt }}\right)^{\dagger}$. We stress that the parameter $k$ here can take any real value and hence this approach can be used to implement noninteger and negative powers of $U$.

One important application of variational unitary diagonalization is quantum simulation. Let $U$ be a Trotterized (or other) approximation to a short-time evolution $e^{-i H \Delta t}$ by some time-independent Hamiltonian $H$. We assume that $H$ is local [46-49], sparse [50-52], or given by a linear combination of unitaries [53-55], permitting efficient simulation with bounded error. Then $W D^{t / \Delta t} W^{\dagger}$ is an approximate fast-forwarded evolution operator with a circuit depth that is independent of $t$. By contrast, most of the best-known Hamiltonian simulation algorithms [49,52,55] have depths scaling at least linearly in $t$, inhibiting long time simulations on near-term hardware. Fast-forwarding can be applied to commuting time-dependent Hamiltonians of the form $f(t) \cdot H$ as well; in this case, after learning $e^{-i H \Delta \tau}=W D W^{\dagger}$ with a small $\Delta \tau$, we use $\exp \left(-i H \int_{0}^{t} f d t^{\prime}\right)=W D^{\tau(t) / \Delta \tau} W^{\dagger}$, where $\tau(t)=\int_{0}^{t} f d t^{\prime}$ and $\Delta \tau=\tau(\Delta t)$.

This low-depth algorithm, called variational fastforwarding [56], lies at an exciting intersection of machine learning and quantum simulation and is a promising approach in the burgeoning field of variational quantum simulation [57-68]. Variational fast-forwarding has been demonstrated on $1+1$ qubits [56]. Refinements of variational fast-forwarding for simulating a given fixed initial state [67] and for learning the spectral decomposition of a given Hamiltonian [68] have also been proposed. It is important to note that the unitary being diagonalized need not already be known. Therefore, variational unitary diagonalization could also be used to perform a "black box diagonalization" of the dynamics of an unknown experimental system. Thus, this approach provides an algorithmic tool for probing dynamics in an experimental setting.

In this work, we use ibmq_bogota to demonstrate the successful learning of a spectral decomposition on $2+2$ qubits. Specifically, we diagonalize the short time evolution unitary for an Ising spin chain. After only 16 steps of training by gradient descent, the spectral decomposition is used to fast forward the evolution of the Ising model, resulting in a dramatic reduction of simulation error compared with Trotterized Hamiltonian simulation and a significant $(\approx 10 \times)$ increase in the effective quantum volume of the simulation. Information
TABLE I. Ising model parameters.

\begin{tabular}{lcc}
\hline \hline & Parameter & Value \\
\hline$N$ & Number spins & 2 \\
$\Delta t$ & Short evolution time & 0.2 \\
$J$ & Exchange energy & 1 \\
$B$ & Transverse field & 1 \\
\hline \hline
\end{tabular}

about the qubits used in the experiment are provided in Appendix A.

\section{METHODS}

\section{A. Learning task}

We demonstrate the variational learning of the spectral decomposition of a unitary by learning a diagonalization of the short-time evolution operator of the two-spin Ising model

$$
H=J \sum_{i=1} Z_{i} Z_{i+1}+B \sum_{i=1,2} X_{i}
$$

Here $J$ quantifies the exchange energy, $B$ is the transverse field strength, and $Z_{i}$ and $X_{i}$ are Pauli operators on the $i$ th qubit. We approximate the short-time evolution $\exp (-i H \Delta t)$ of the spin chain using a two-step, second-order Trotter approximation; that is, we take

$$
U=\left[\prod_{i} R_{\mathrm{x}}\left(\theta_{\mathrm{B}}\right)_{i} \times \prod_{i} R_{\mathrm{zz}}\left(\theta_{\mathrm{J}}\right)_{i, i+1} \times \prod_{i} R_{\mathrm{x}}\left(\theta_{\mathrm{B}}\right)_{i}\right]^{2},
$$

where $\theta_{\mathrm{B}}=B \Delta t / 2$ and $\theta_{\mathrm{J}}=J \Delta t$. The simulated Ising model parameters are listed in Table I. The specific circuit we used for $U$ is shown in Fig. 1.

\section{B. Ansatz}

To learn the spectral decomposition of $U$, we variationally compile it to a unitary with a structure of the form

$$
V(\boldsymbol{\theta}, \boldsymbol{\gamma})=W(\boldsymbol{\theta}) D(\boldsymbol{\gamma}) W(\boldsymbol{\theta})^{\dagger},
$$

where $W$ is an arbitrary unitary, $D$ is a diagonal matrix, and $\boldsymbol{\theta}$ and $\boldsymbol{\gamma}$ are vectors of parameters. After successful training, $D$ will capture the eigenvalues of $U$, and $W$ will capture the rotation matrix from the computational basis to the eigenbasis of $U$.

The parameterized circuits we used as ansatzes for the diagonal unitary $D$ and basis transformation $W$ are shown in Fig. 2. A general diagonal unitary $D \in \mathrm{SU}\left(2^{n}\right)$ on $n$ qubits contains $2^{n}-1$ variational parameters. In our experiment, we implement a two-qubit version of this exact $D$ containing three variables. In general, an arbitrary unitary $W$ can be constructed from any general $n$-qubit parameterized quantum circuit with poly $(n)$ variables. The expressive power of different options has been investigated in Refs. [69,70]. The two-qubit circuit we use to implement the arbitrary unitary $W$ consists of three layers of $X, Y$ rotations and phase gates on each qubit, separated by two CNOT gates. 


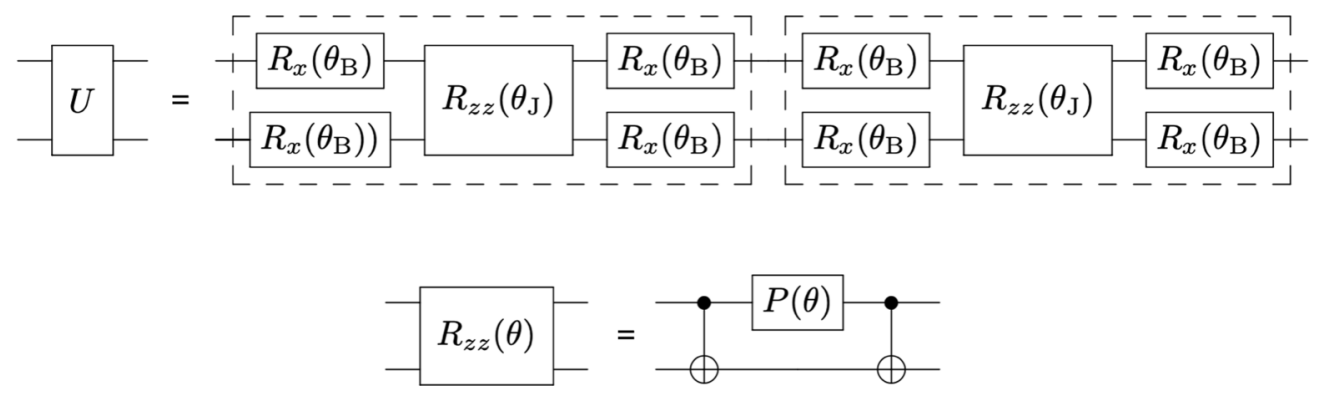

FIG. 1. Circuit for the short-time evolution of the Ising model. The boxes indicate individual Trotter steps. Here $R_{\mathrm{x}}(\theta)=e^{-i \theta X / 2}$ is a single-qubit Pauli $X$ rotation, $P(\gamma)=\operatorname{diag}\left(1, e^{i \gamma}\right)$ is a single-qubit phase gate, and $R_{\mathrm{zz}}(\theta)=e^{-i \theta Z \otimes Z / 2}$ is a two-qubit diagonal unitary.

\section{Cost function}

To compile the target unitary into a diagonal form, we use the local Hilbert-Schmidt test cost function defined in Ref. [38], which can be written as

$$
C=1-\frac{1}{n} \sum_{i=1}^{n} \operatorname{Pr}(00)_{A_{i}, B_{i}},
$$

where $\operatorname{Pr}(00)_{A_{i}, B_{i}}$ is the probability of observing outcome 00 on the pair of qubits $\left(A_{i}, B_{i}\right)$ from registers $A$ and $B$; see Fig. 3.

The probabilities $\left\{\operatorname{Pr}(00)_{A_{i}, B_{i}}\right\}_{i=1}^{n}$ are measures of the entanglement fidelity of the unitary channel $U V^{\dagger}$ with $V=$ $W D W^{\dagger}$. As a result, this cost is faithful, vanishing if and only if the diagonalization $W D W^{\dagger}$ matches the target unitary $U$ (up to a global phase). Furthermore, the cost is operationally meaningful for nonzero values in virtue of upper bounding the average gate fidelity between $U$ and $V$. Hence, a small value of $C$ guarantees that the diagonalization $W D W^{\dagger}$ is an accurate approximation of the target unitary $U$. We note that the cost (4) involves only local measures and hence mitigates trainability issues associated with barren plateaus [71-82]. For learning a $4 \times 4$ unitary matrix,

$$
C=1-\frac{1}{2}\left[\operatorname{Pr}(00)_{1,2}+\operatorname{Pr}(00)_{3,4}\right],
$$

where $\operatorname{Pr}(00)_{1,2}$ and $\operatorname{Pr}(00)_{3,4}$ are the 00-state probabilities measured after running the circuits shown in Figs. 4(a) and 4(b), respectively. For more details on the cost function, see Ref. [38].

\section{Training}

The parameters $\left(\theta_{1}, \ldots, \theta_{18}, \gamma_{1}, \ldots, \gamma_{3}\right)$ are initialized to minimize the cost (5) with the exchange energy $J$ in (1) set to zero, which can be simulated efficiently and solved exactly. This procedure reduces the chance of the optimization leading to a local but not global minimum of the cost, as $C$ is not convex.

The circuits are trained to minimize the cost using gradient descent. At each step of the training, we measure the cost and gradients $\frac{\partial C}{\partial \theta_{k}}$ and $\frac{\partial C}{\partial \gamma_{l}}$ at a particular point $(\boldsymbol{\theta}, \boldsymbol{\gamma})$ in the parameter space and use this to update $(\boldsymbol{\theta}, \boldsymbol{\gamma})$ according to

$$
\begin{gathered}
\theta_{k} \leftarrow \theta_{k}-\eta \frac{\partial C}{\partial \theta_{k}}, \\
\gamma_{l} \leftarrow \gamma_{l}-\eta \frac{\partial C}{\partial \gamma_{l}},
\end{gathered}
$$

where $\eta$ is the learning rate. The gradients $\frac{\partial C}{\partial \theta_{k}}$ and $\frac{\partial C}{\partial \gamma_{l}}$ are measured using the parameter shift rule derived in Ref. [56]. In each optimization step, the 21 components of the gradient are measured, requiring the measurement of 156 distinct circuits. For each cost evaluation, we took 8000 measurement shots, close to the maximum supported by the back end without incurring additional circuit overhead

The learning rate was decreased as the optimization progressed according to the schedule

$$
\eta(j)=\frac{\eta_{0}}{[1+(j / \delta)]^{\kappa}},
$$

where $j \in\{0,1,2, \ldots, 16\}$ is the optimization step number. The hyperparameters $\eta_{0}=1.1, \kappa=0.5$, and $\delta=12$ were optimized by classical simulation. Additional details about the training are provided in Appendix B.

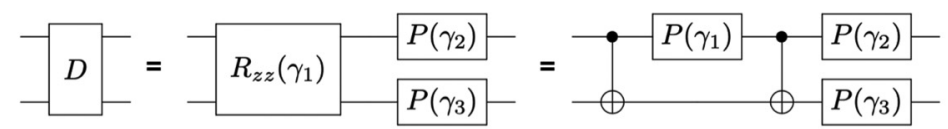

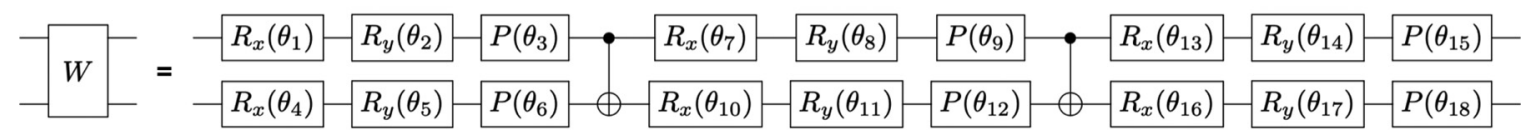

FIG. 2. Ansatz circuits. Implemented circuits for the diagonal unitary $D$ and basis transformation $W$. Here $R_{\mathrm{zz}}(\gamma)=e^{-i \gamma Z \otimes Z / 2}$ is a two-qubit diagonal unitary and $P(\gamma)=\operatorname{diag}\left(1, e^{i \gamma}\right)$ is a single-qubit phase gate. $D$ contains three variational parameters. $W$ has three layers and 18 variational parameters. 


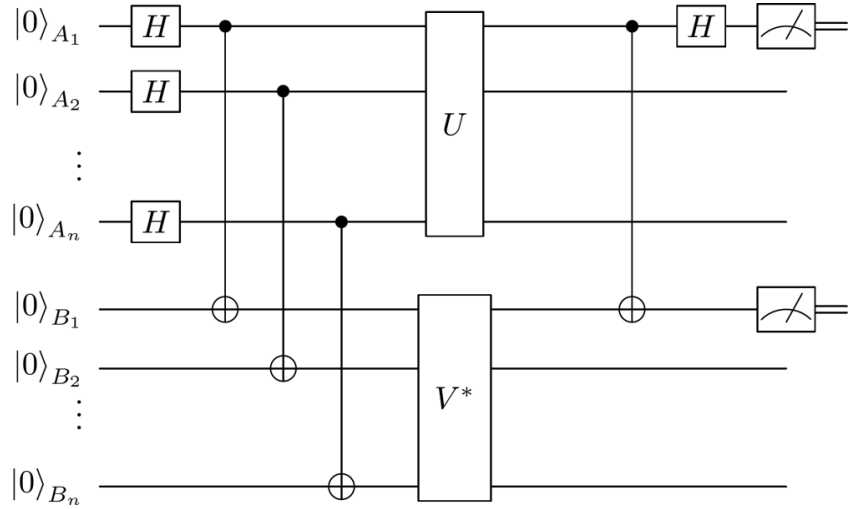

FIG. 3. Circuit for the learning of $U$ by a variational circuit $V$. The input $U$ can be known to the user and provided in the form of an efficient circuit construction, or unknown but provided by another algorithm. Measurements are performed on pairs of qubits $\left(A_{i}, B_{i}\right)$ from registers $A$ and $B$.

\section{RESULTS}

To assess the quality of the optimization, the parameters found at each step of the training were used to evaluate $C$ both on the quantum computer and classically. The results are shown in Fig. 5. The classical cost, which we call the noise-free or ideal cost, reflects the true accuracy of the optimized circuits. We successfully reduced the raw cost to 0.110 corresponding to an ideal cost of 0.013 . The raw cost from the quantum computer is higher than the ideal cost because of gate errors.

The inset of Fig. 5 confirms that the errors in $D$ and $W D W^{\dagger}$ are both iteratively reduced as the cost is trained. Here the Frobenius distance between $U$ and $V$ is plotted, minimized over the arbitrary global phase $e^{i \varphi}$. The ideal and learned diagonals are also compared, accounting for the global phase $e^{i \varphi}$ and for a possible reordering, specified by a permutation matrix $\chi$. Specifically, we plot the Frobenius distance $\min _{\varphi, \chi}\left\|D_{\text {exact }}-e^{i \varphi} \chi D \chi^{\dagger}\right\|$, where $D_{\text {exact }}$ is a diagonal matrix with $\left\{\lambda_{i}^{\text {exact }}\right\}$, the ordered exact spectrum of $U$, along

(a)

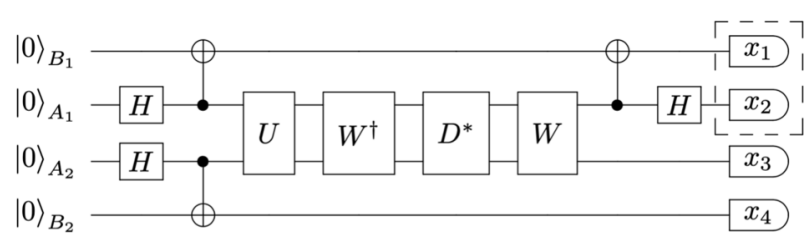

(b)

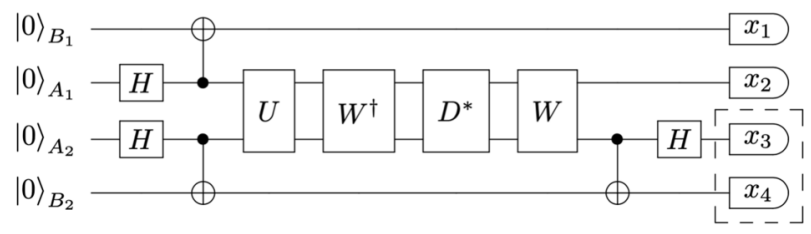

FIG. 4. Cost function circuit. Circuits to learn the spectral decomposition $W D W^{\dagger}$ of a two-qubit unitary $U$ on a four-qubit chain. Each cost function and gradient evaluation requires the estimation of $\operatorname{Pr}(00)_{1,2}$ and $\operatorname{Pr}(00)_{3,4}$, obtained by measuring the qubits in the dashed boxes of panels (a) and (b).

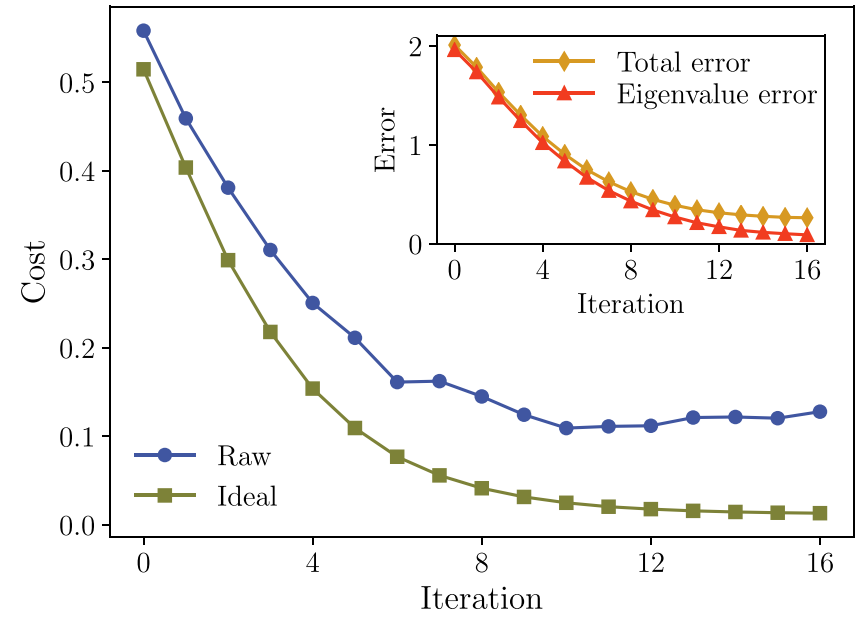

FIG. 5. Training curves. In the main plot, we show the cost as a function of the iteration step during training. The blue curve is the measured cost function $C$ and the green curve is its noise-free "ideal" value. In the inset, the yellow curve indicates the total error in the learned unitary defined as Frobenius distances between $U$ and $V$ minimized over a global phase $e^{i \varphi}$, i.e., the quantity $\min _{\varphi, \chi}\left\|U-e^{i \varphi} V\right\|$. The red curve indicates the eigenvalue error, defined as the Frobenius distance between the learned and exact diagonal also minimized over a permutation $\chi$, i.e., $\min _{\varphi, \chi}\left\|D_{\text {exact }}-e^{i \varphi} \chi D \chi \chi^{\dagger}\right\|$.

the diagonal. This is equivalent to the sum of the eigenvalue errors $\sum_{i}\left|\lambda_{i}^{\text {exact }}-\lambda_{i} e^{i \varphi_{\text {opt }}}\right|^{2}$, where $\left\{\lambda_{i}\right\}$ is the ordered learned spectrum and $\varphi_{\mathrm{opt}}$ accounts for the global phase shift.

It is also interesting to monitor the training by using the measured gradient $\boldsymbol{g}_{\text {meas }}$ at each step to calculate the angle between $\boldsymbol{g}_{\text {meas }}$ and the exact gradient simulated classically. This is plotted in Appendix B. The data confirm that the optimization is correctly moving downhill in the cost function landscape.

Having learned the spectral decomposition, we use the result to fast forward the Hamiltonian simulation of the Ising model (1) for two spins on ibmq_bogota. In this experiment, we prepare an initial state $|+\rangle^{\otimes 2}$ and propagate it forward in time by both Trotterization and variational fast forwarding (with back-end circuit optimization disabled in both cases). The Trotterized evolution at times $t=K \Delta t$ is obtained by applying $K=0,8,16, \ldots, 96$ consecutive Trotter steps from Fig. 1. After each step, we experimentally measure the fidelity of the Trotterized evolution with the perfect evolution $e^{-i H t}|+\rangle^{\otimes 2}$, which contains no Trotter error. The variational fast-forwarding evolution at time $t$ is obtained by applying the optimized variational circuits for $W^{\dagger}, D$, and $W$ to $|+\rangle^{\otimes 2}$, but with $D$ 's parameters $\left(\gamma_{1}, \gamma_{2}, \gamma_{3}\right)$ changed to

$$
\left(\gamma_{1}, \gamma_{2}, \gamma_{3}\right) \frac{t}{\Delta t} \text {. }
$$

The state fidelity with perfect evolution $e^{-i H t}|+\rangle^{\otimes 2}$ is also measured in this case. The results for this experimental fast forwarding and experimental Trotter simulation are indicated by the green and blue solid lines respectively in Fig. 6 .

We compare the experimental fast forwarding to the ideal classical fast forwarding by also measuring the noisefree fidelities obtained by classical simulation. In this ideal 


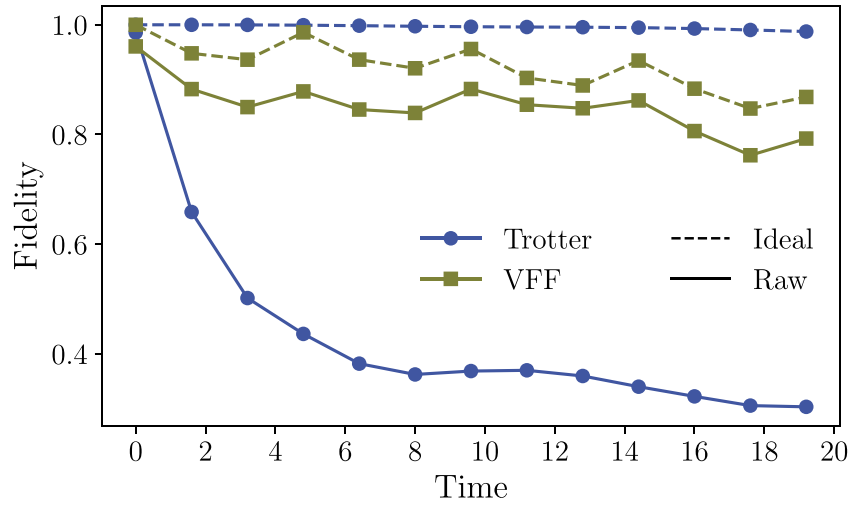

FIG. 6. Demonstration of variational fast forwarding. The plot shows the measured state fidelity vs evolution time. The data (circles) are obtained at multiples of $8 \Delta t$, out to $t=19.2$.

simulation, the initial state $|+\rangle^{\otimes 2}$ is prepared perfectly and the Trotterized evolution includes Trotter errors but no gate errors. The measurement is also assumed to be ideal. The fidelities of these ideal simulations are indicated by the dashed lines in Fig. 6.

The ideal Trotterized evolution is nearly perfect in Fig. 6 due to the small value of $\Delta t$ and use of a second-order Trotter step. The ideal variational fast-forwarding evolution is less accurate, due to the imperfect learning of $U$ 's spectral decomposition (because noise during the learning prevents even the ideal cost, the green curve in Fig. 5, to reach zero). However, the real data taken on ibmq_bogota exhibit the opposite behavior: Whereas the variational fast-forwarding evolution is only slightly degraded by gate errors-because the circuit depth is independent of $t$ - the Trotterized evolution quickly loses fidelity. Namely, while the fidelity of the variationally fast-forwarded simulation remains above 0.7 for all 20 times steps, the fidelity of the Trotterized simulation is less than 0.4 after only six times steps. Thus, Fig. 6 demonstrates a striking example of fast forwarding as well as provides further evidence that the spectral decomposition of $U$ has been well learned.

In conclusion, we have experimentally demonstrated the entanglement-enhanced quantum learning of a $4 \times 4$ unitary using a diagonal ansatz containing a total of six CNOTs and 21 variational parameters. A single input-output pair was used for training compared to the $2^{2}=4$ input-output pairs necessary for training without entangling ancillas. We both learn the unitary and its spectral decomposition, enabling the fast forwarding of an Ising model. This four-qubit experiment took 8000 shots for each of the 156 independent circuit evaluations, at each of the 16 steps of the optimization algorithm, for a total of $\approx 2500$ distinct circuit implementations. It is among the most complex quantum machine learning demonstrations

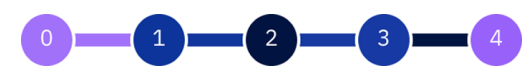

FIG. 7. Layout of IBM Q device ibmq_bogota. In this work, we use qubits $Q_{0}, Q_{1}, Q_{2}$, and $Q_{3}$ for the training and qubits $Q_{1}$ and $Q_{2}$ for the Trotter and VFF comparison.
TABLE II. Calibration data provided by IBM Q for the ibmq_bogota chip during the period of data acquisition.

\begin{tabular}{lcccc}
\hline \hline Qubit & $T_{1}(\mu s)$ & $T_{2}(\mu s)$ & SPAM error $\epsilon$ & $U_{2}$ error \\
\hline$Q_{0}$ & 136.6 & 178.0 & 0.056 & $3.70 \times 10^{-4}$ \\
$Q_{1}$ & 132.3 & 117.4 & 0.045 & $2.01 \times 10^{-4}$ \\
$Q_{2}$ & 65.4 & 132.4 & 0.024 & $2.48 \times 10^{-4}$ \\
$Q_{3}$ & 103.6 & 158.3 & 0.020 & $4.74 \times 10^{-4}$ \\
\multicolumn{5}{c}{ CNOT gates } \\
CNOT $_{0,1}$ & CNOT $_{1,0}$ & CNOT error \\
$\mathrm{CNOT}_{1,2}$ & \multicolumn{5}{c}{$\mathrm{CNOT}_{2,1}$} & $1.04 \times 10^{-3}$ \\
$\mathrm{CNOT}_{2,3}$ & \multicolumn{5}{c}{} & $8.12 \times 10^{-3}$ \\
\hline \hline
\end{tabular}

to date [14-20] and constitutes an important primitive in experimental quantum information science.

\section{ACKNOWLEDGMENTS}

P.J.C. and A.S. acknowledge initial support and M.R.G. acknowledges support from LANL's Laboratory Directed Research and Development (LDRD) program under Project No. 20190065DR. Additionally, P.J.C. and A.S. acknowledge that (subsequent to the above acknowledged funding) this material is based upon work supported by the U.S. Department of Energy, Office of Science, National Quantum Information Science Research Centers. Z.H. acknowledges support from the LANL ASC Beyond Moore's Law project. We would also like to thank Andrew Arrasmith, Lukasz Cincio, and Joe Gibbs for useful discussions.

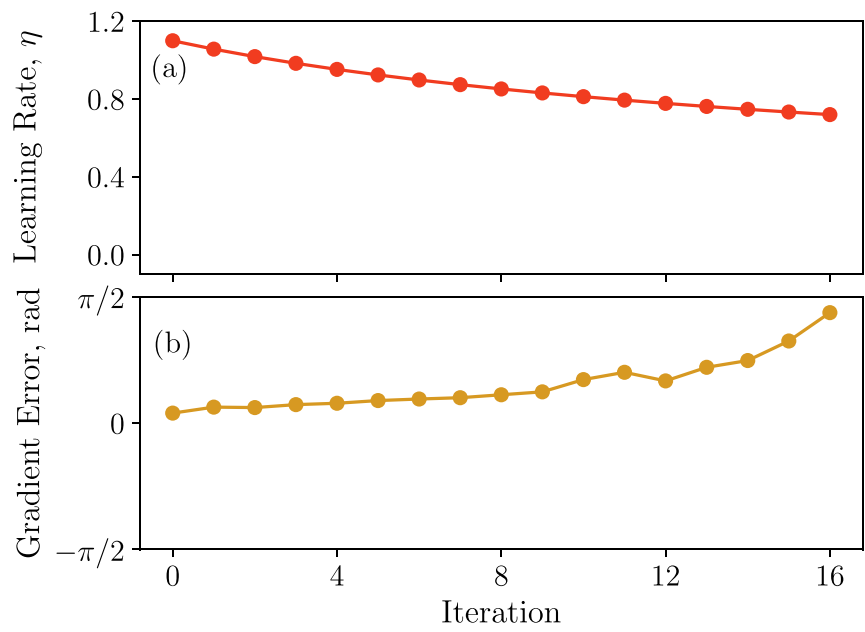

FIG. 8. (a) Learning rate vs optimization step during training by gradient descent. This rate obeys $\eta(j)=\eta_{0} /[1+(j / \delta)]^{\kappa}$, where $j \in\{0,1,2, \ldots, 16\}$ is the optimization step number and the hyperparameters $\eta_{0}=1.1, \kappa=0.5$, and $\delta=12$ were optimized by classical simulation. (b) Error in the measured gradient direction during training. Here we plot the angle between gradient measured on ibmq_bogota during training, and the true gradient calculated classically for the same set of parameters, for each optimization step. 


\section{APPENDIX A: QUBITS}

Data were taken on the IBM Q processor ibmq_bogota using the BPQ software package developed by Geller and colleagues. BPQ is a PYTHON package developed to design, run, and analyze complex quantum computing and quantum information experiments using commercial back ends. We demonstrate the learning of a spectral decomposition using the qubits shown in Fig. 7. Calibration data supplied by the back end are summarized in Tables II(a) and II(b).

Here $T_{1,2}$ are the standard Markovian decoherence times, and

$$
\epsilon=\frac{T(0 \mid 1)+T(1 \mid 0)}{2}
$$

is the single-qubit state-preparation and measurement (SPAM) error, averaged over initial states. The $U_{2}$ error column gives the single-qubit gate error measured by randomized benchmarking. The CNOT errors are also measured by randomized benchmarking.

\section{APPENDIX B: TRAINING}

The variational circuits for $W$ and $D$ were trained by gradient descent

$$
\begin{gathered}
\theta_{k} \leftarrow \theta_{k}-\eta \frac{\partial C_{\mathrm{LHST}}}{\partial \theta_{k}} \\
\gamma_{l} \leftarrow \gamma_{l}-\eta \frac{\partial C_{\mathrm{LHST}}}{\partial \gamma_{l}},
\end{gathered}
$$

where $\theta_{k}$ and $\gamma_{l}$ are the variational parameters for the $W$ and $D$ circuits, respectively, and $\eta$ is the learning rate. We used the variable learning rate plotted in Fig. 8(a), which was optimized by classical simulation. At each step of the training, we measure the cost $C_{\mathrm{LHST}}$ and gradients $\partial C_{\mathrm{LHST}} / \partial \theta_{k}$ and $\partial C_{\mathrm{LHST}} / \partial \gamma_{l}$ at a particular point $\left(\theta_{1}, \ldots, \theta_{18}, \gamma_{1}, \ldots, \gamma_{3}\right)$ in parameter space, and use this to update $\left(\theta_{1}, \ldots, \theta_{18}, \gamma_{1}, \ldots, \gamma_{3}\right)$ according to (B1). We also calculate the angle between the measured gradient and the exact gradient simulated classically, which is plotted in Fig. 8(b). These data show that the measured gradient is correctly pointing uphill until the end of the training when a local minimum is reached and the gradient becomes small and noisy.
[1] J. Biamonte, P. Wittek, N. Pancotti, P. Rebentrost, N. Wiebe, and S. Lloyd, Quantum machine learning, Nature (London) 549, 195 (2017).

[2] A. W. Harrow, A. Hassidim, and S. Lloyd, Quantum Algorithm for Linear Systems of Equations, Phys. Rev. Lett. 103, 150502 (2009).

[3] B. D. Clader, B. C. Jacobs, and C. R. Sprouse, Preconditioned Quantum Linear System Algorithm, Phys. Rev. Lett. 110, 250504 (2013).

[4] A. M. Childs, R. Kothari, and R. D. Somma, Quantum algorithm for systems of linear equations with exponentially improved dependence on precision, SIAM J. Comput. 46, 1920 (2017).

[5] M. Schuld and N. Killoran, Quantum Machine Learning in Feature Hilbert Spaces, Phys. Rev. Lett. 122, 040504 (2019).

[6] V. Havlíček, A. D. Córcoles, K. Temme, A. W. Harrow, A. Kandala, J. M. Chow, and J. M. Gambetta, Supervised learning with quantum-enhanced feature spaces, Nature (London) 567, 209 (2019)

[7] N. Wiebe, A. Kapoor, and K. M. Svore, Quantum deep learning, arXiv:1412.3489.

[8] G. Verdon, M. Broughton, and J. Biamonte, A quantum algorithm to train neural networks using low-depth circuits, arXiv:1712.05304.

[9] A. Kandala, A. Mezzacapo, K. Temme, M. Takita, M. Brink, J. M. Chow, and J. M. Gambetta, Hardware-efficient variational quantum eigensolver for small molecules and quantum magnets, Nature (London) 549, 242 (2017).

[10] A. Kandala, K. Temme, A. D. Córcoles, A. Mezzacapo, J. M. Chow, and J. M. Gambetta, Error mitigation extends the computational reach of a noisy quantum processor, Nature (London) 567, 491 (2019).

[11] F. Arute, K. Arya, R. Babbush, D. Bacon, J. C. Bardin, R. Barends, S. Boixo, M. Broughton, B. B. Buckley, D. A. Buell,
B. Burkett, N. Bushnell, Yu Chen, Z. Chen, B. Chiaro, R. Collins, W. Courtney, S. Demura, A. Dunsworth et al. (Google AI Quantum and Collaborators), Hartree-Fock on a superconducting qubit quantum computer, Science 369, 1084 (2020).

[12] F. Arute, K. Arya, R. Babbush, D. Bacon, J. C. Bardin, R. Barends, A. Bengtsson, S. Boixo, M. Broughton, B. B. Buckley et al., Observation of separated dynamics of charge and spin in the Fermi-Hubbard model, arXiv:2010.07965.

[13] C. Neill, T. McCourt, X. Mi, Z. Jiang, M. Y. Niu, W. Mruczkiewicz, I. Aleiner, F. Arute, K. Arya, J. Atalaya et al., Accurately computing the electronic properties of a quantum ring, Nature (London) 594, 508 (2021).

[14] D. Riste, M. P. da Silva, C. A. Ryan, A. W. Cross, A. D. Córcoles, J. A. Smolin, J. M. Gambetta, J. M. Chow, and B. R. Johnson, Demonstration of quantum advantage in machine learning, npj Quantum Inf. 3, 16 (2017).

[15] M. Schuld, M. Fingerhuth, and F. Petruccione, Implementing a distance-based classifier with a quantum interference circuit, EPL 119, 60002 (2017).

[16] X.-W. Yao, H. Wang, Z. Liao, M.-C. Chen, J. Pan, J. Li, K. Zhang, X. Lin, Z. Wang, Z. Luo et al., Quantum Image Processing and its Application to Edge Detection: Theory and Experiment, Phys. Rev. X 7, 031041 (2017).

[17] H.-L. Huang, X.-L. Wang, P. P. Rohde, Y.-H. Luo, Y.-W. Zhao, C. Liu, L. Li, N.-L. Liu, C.-Y. Lu, and J.-W. Pan, Demonstration of topological data analysis on a quantum processor, Optica $\mathbf{5}$, 193 (2018).

[18] D. Zhu, N. M. Linke, M. Benedetti, K. A. Landsman, N. H. Nguyen, C. H. Alderete, A. Perdomo-Ortiz, N. Korda, A. Garfoot, C. Brecque, L. Egan, O. Perdomo, and C. Monroe, Training of quantum circuits on a hybrid quantum computer, Sci. Adv. 5, eaaw9918 (2019).

[19] K. Kathuria, A. Ratan, M. McConnell, and S. Bekiranov, Implementation of a Hamming distance-like genomic quantum 
classifier using inner products on ibmqx2 and ibmq_16_melbourne, Quantum Machine Intell. 2, 7 (2020).

[20] T. Tomesh, P. Gokhale, E. R. Anschuetz, and F. T. Chong, Core set clustering on small quantum computers, arXiv:2004.14970.

[21] M. Cerezo, A. Arrasmith, R. Babbush, S. C. Benjamin, S. Endo, K. Fujii, J. R. McClean, K. Mitarai, X. Yuan, L. Cincio, and P. J. Coles, Variational quantum algorithms, arXiv:2012.09265.

[22] S. Endo, Z. Cai, S. C. Benjamin, and X. Yuan, Hybrid quantumclassical algorithms and quantum error mitigation, J. Phys. Soc. Jpn. 90, 032001 (2021).

[23] K. Bharti, A. Cervera-Lierta, T. H. Kyaw, T. Haug, S. Alperin-Lea, A. Anand, M. Degroote, H. Heimonen, J. S. Kottmann, T. Menke, W.-K. Mok, S. Sim, L.-C. Kwek, and A. Aspuru-Guzik, Noisy intermediate-scale quantum algorithms, arXiv:2101.08448.

[24] J. Romero, J. P. Olson, and A. Aspuru-Guzik, Quantum autoencoders for efficient compression of quantum data, Quantum Sci. Technol. 2, 045001 (2017).

[25] D. Bondarenko and P. Feldmann, Quantum Autoencoders to Denoise Quantum Data, Phys. Rev. Lett. 124, 130502 (2020).

[26] C. Bravo-Prieto, R. LaRose, M. Cerezo, Y. Subasi, L. Cincio, and P. J. Coles, Variational quantum linear solver, arXiv:1909.05820.

[27] X. Xu, J. Sun, S. Endo, Y. Li, S. C. Benjamin, and X. Yuan, Variational algorithms for linear algebra, Sci. Bull. (2021), doi: 10.1016/j.scib.2021.06.023.

[28] H.-Y. Huang, K. Bharti, and P. Rebentrost, Near-term quantum algorithms for linear systems of equations, arXiv:1909.07344.

[29] A. Peruzzo, J. McClean, P. Shadbolt, M.-H. Yung, X.-Q. Zhou, P. J. Love, A. Aspuru-Guzik, and J. L. O'brien, A variational eigenvalue solver on a photonic quantum processor, Nat. Commun. 5, 4213 (2014).

[30] E. Farhi, J. Goldstone, and S. Gutmann, A quantum approximate optimization algorithm, arXiv:1411.4028.

[31] S. Hadfield, Z. Wang, B. O'Gorman, E. G. Rieffel, D. Venturelli, and R. Biswas, From the quantum approximate optimization algorithm to a quantum alternating operator ansatz, Algorithms 12, 34 (2019).

[32] K. Mitarai, M. Negoro, M. Kitagawa, and K. Fujii, Quantum circuit learning, Phys. Rev. A 98, 032309 (2018).

[33] R. LaRose, A. Tikku, É. O’Neel-Judy, L. Cincio, and P. J. Coles, Variational quantum state diagonalization, npj Quantum Inf. 5, 57 (2019).

[34] M. Cerezo, K. Sharma, A. Arrasmith, and P. J. Coles, Variational quantum state eigensolver, arXiv:2004.01372.

[35] C. Bravo-Prieto, D. García-Martín, and J. I. Latorre, Quantum singular value decomposer, Phys. Rev. A 101, 062310 (2020).

[36] M. Cerezo, A. Poremba, L. Cincio, and P. J. Coles, Variational quantum fidelity estimation, Quantum 4, 248 (2020).

[37] J. L. Beckey, M. Cerezo, A. Sone, and P. J. Coles, Variational quantum algorithm for estimating the quantum Fisher information, arXiv:2010.10488.

[38] S. Khatri, R. LaRose, A. Poremba, L. Cincio, A. T. Sornborger, and P. J. Coles, Quantum-assisted quantum compiling, Quantum 3, 140 (2019).

[39] K. Sharma, S. Khatri, M. Cerezo, and P. J. Coles, Noise resilience of variational quantum compiling, New J. Phys. 22, 043006 (2020).

[40] T. Jones and S. C. Benjamin, Quantum compilation and circuit optimisation via energy dissipation, arXiv:1811.03147.
[41] L. Cincio, Y. Subaş1, A. T. Sornborger, and P. J. Coles, Learning the quantum algorithm for state overlap, New J. Phys. 20, 113022 (2018).

[42] M. Horodecki, P. Horodecki, and R. Horodecki, General teleportation channel, singlet fraction, and quasidistillation, Phys. Rev. A 60, 1888 (1999).

[43] M. A. Nielsen, A simple formula for the average gate fidelity of a quantum dynamical operation, Phys. Lett. A 303, 249 (2002).

[44] K. Poland, K. Beer, and T. J. Osborne, No free lunch for quantum machine learning, arXiv:2003.14103.

[45] K. Sharma, M. Cerezo, Z. Holmes, L. Cincio, A. Sornborger, and P. J. Coles, Reformulation of the no-free-lunch theorem for entangled data sets, arXiv:2007.04900.

[46] S. Lloyd, Universal quantum simulators, Science 273, 1073 (1996).

[47] A. M. Childs and Y. Su, Nearly Optimal Lattice Simulation by Product Formulas, Phys. Rev. Lett. 123, 050503 (2019).

[48] J. Haah, M. B. Hastings, R. Kothari, and G. H. Low, Quantum algorithm for simulating real time evolution of lattice Hamiltonians, SIAM J. Comput., 350 (2021).

[49] G. H. Low, V. Kliuchnikov, and N. Wiebe, Well-conditioned multiproduct Hamiltonian simulation, arXiv:1907.11679.

[50] D. Aharonov and A. Ta-Shma, Adiabatic quantum state generation and statistical zero knowledge, in Proceedings of the Thirty-fifth Annual ACM Symposium on Theory of Computing (ACM, 2003), pp. 20-29.

[51] D. W. Berry, A. M. Childs, R. Cleve, R. Kothari, and R. D. Somma, Exponential improvement in precision for simulating sparse Hamiltonians, in Proceedings of the Forty-sixth Annual ACM Symposium on Theory of Computing (ACM, 2014), pp. 283-292.

[52] G. H. Low and I. L. Chuang, Optimal Hamiltonian Simulation by Quantum Signal Processing, Phys. Rev. Lett. 118, 010501 (2017).

[53] D. W. Berry, A. M. Childs, R. Cleve, R. Kothari, and R. D. Somma, Simulating Hamiltonian Dynamics with a Truncated Taylor Series, Phys. Rev. Lett. 114, 090502 (2015).

[54] D. W. Berry, A. M. Childs, and R. Kothari, Hamiltonian simulation with nearly optimal dependence on all parameters, in 2015 IEEE 56th Annual Symposium on Foundations of Computer Science (IEEE, 2015), pp. 792-809.

[55] G. H. Low and I. L. Chuang, Hamiltonian simulation by qubitization, Quantum 3, 163 (2019).

[56] C. Cîrstoiu, Z. Holmes, J. Iosue, L. Cincio, P. J. Coles, and A. Sornborger, Variational fast forwarding for quantum simulation beyond the coherence time, npj Quantum Inf. 6, 82 (2020).

[57] A. Arrasmith, L. Cincio, A. T. Sornborger, W. H. Zurek, and P. J. Coles, Variational consistent histories as a hybrid algorithm for quantum foundations, Nat. Commun. 10, 3438 (2019).

[58] C. J. Trout, M. Li, M. Gutiérrez, Y. Wu, S.-T. Wang, L. Duan, and K. R. Brown, Simulating the performance of a distance3 surface code in a linear ion trap, New J. Phys. 20, 043038 (2018).

[59] S. Endo, J. Sun, Y. Li, S. C. Benjamin, and X. Yuan, Variational Quantum Simulation of General Processes, Phys. Rev. Lett. 125, 010501 (2020).

[60] Y.-X. Yao, N. Gomes, F. Zhang, C.-Z. Wang, K.-M. Ho, T. Iadecola, and P. P. Orth, Adaptive variational quantum dynamics simulations, PRX Quantum 2, 030307 (2021). 
[61] M. Benedetti, M. Fiorentini, and M. Lubasch, Hardwareefficient variational quantum algorithms for time evolution, Phys. Rev. Research 3, 033083 (2021).

[62] K. Heya, K. M. Nakanishi, K. Mitarai, and K. Fujii, Subspace variational quantum simulator, arXiv:1904.08566.

[63] K. Bharti and T. Haug, Quantum assisted simulator, arXiv:2011.06911.

[64] J. W. Z. Lau, K. Bharti, T. Haug, and L. C. Kwek, Quantum assisted simulation of time dependent Hamiltonians, arXiv:2101.07677.

[65] T. Haug and K. Bharti, Generalized quantum assisted simulator, arXiv:2011.14737.

[66] S. Barison, F. Vicentini, and G. Carleo, An efficient quantum algorithm for the time evolution of parameterized circuits, Quantum 5, 512 (2021).

[67] J. Gibbs, K. Gili, Z. Holmes, B. Commeau, A. Arrasmith, L. Cincio, P. J. Coles, and A. Sornborger, Long-time simulations with high fidelity on quantum hardware, arXiv:2102.04313.

[68] B. Commeau, M. Cerezo, Z. Holmes, L. Cincio, P. J. Coles, and A. Sornborger, Variational Hamiltonian diagonalization for dynamical quantum simulation, arXiv:2009.02559.

[69] J. Tangpanitanon, S. Thanasilp, N. Dangniam, M.-A. Lemonde, and D. G. Angelakis, Expressibility and trainability of parameterized analog quantum systems for machine learning applications, Phys. Rev. Research 2, 043364 (2020).

[70] S. Sim, P. D. Johnson, and A. Aspuru-Guzik, Expressibility and entangling capability of parameterized quantum circuits for hybrid quantum-classical algorithms, Adv. Quantum Technol. 2, 1900070 (2019).

[71] J. R. McClean, S. Boixo, V. N. Smelyanskiy, R. Babbush, and $\mathrm{H}$. Neven, Barren plateaus in quantum neural network training landscapes, Nat. Commun. 9, 4812 (2018).
[72] M. Cerezo, A. Sone, T. Volkoff, L. Cincio, and P. J. Coles, Cost function dependent barren plateaus in shallow parametrized quantum circuits, Nat. Commun. 12, 1791 (2021).

[73] A. Uvarov and J. Biamonte, On barren plateaus and cost function locality in variational quantum algorithms, J. Phys. A 54 (24), 245301 (2021).

[74] S. Wang, E. Fontana, M. Cerezo, K. Sharma, A. Sone, L. Cincio, and P. J. Coles, Noise-induced barren plateaus in variational quantum algorithms, arXiv:2007.14384.

[75] M. Cerezo and P. J. Coles, Higher order derivatives of quantum neural networks with barren plateaus, Quantum Sci. Technol. 6, 035006 (2021).

[76] A. Pesah, M Cerezo, S. Wang, T. Volkoff, A. T. Sornborger, and P. J. Coles, Absence of barren plateaus in quantum convolutional neural networks, arXiv:2011.02966.

[77] Z. Holmes, A. Arrasmith, B. Yan, P. J. Coles, A. Albrecht, and A. T. Sornborger, Barren Plateaus Preclude Learning Scramblers, Phys. Rev. Lett. 126, 190501 (2021).

[78] A. Arrasmith, M Cerezo, P. Czarnik, L. Cincio, and P. J. Coles, Effect of barren plateaus on gradient-free optimization, arXiv:2011.12245.

[79] C. O. Marrero, M. Kieferová, and N. Wiebe, Entanglement induced barren plateaus, arXiv:2010.15968.

[80] T. L. Patti, K. Najafi, X. Gao, and S. F. Yelin, Entanglement devised barren plateau mitigation, Phys. Rev. Research 3, 033090 (2021).

[81] Z. Holmes, K. Sharma, M Cerezo, and P. J. Coles, Connecting ansatz expressibility to gradient magnitudes and barren plateaus, arXiv:2101.02138.

[82] E. Grant, L. Wossnig, M. Ostaszewski, and M. Benedetti, An initialization strategy for addressing barren plateaus in parametrized quantum circuits, Quantum 3, 214 (2019). 